\title{
Capacidad de Proceso: Una herramienta de decisión Empresarial en el armado de vallas metálicas
}

\section{Process Capacity: A Business Decision Tool in the assembly of metal fences}

DOI: http://dx.doi.org/10.17994/bilo

Artículo de Investigación Científica. Fecha de Recepción: 30/05/2020. Fecha de Aceptación: 14/06/2020

Cristian Navarro-Navarro

Universidad de la Costa CUC, Barranquilla, (Colombia)

Cnavarro@cuc.edu.co

Adelina Gutiérrez

Universidad de la Costa CUC, Barranquilla, (Colombia)

agutierr51@cuc.edu.co

Esteban Sarmiento

Universidad de la Costa CUC, Barranquilla, (Colombia)

esarmien12@cuc.edu.co

Alexander Troncoso-Palacio

Universidad de la Costa CUC, Barranquilla, (Colombia)

atroncos1@ cuc.edu.co

Para citar este artículo:

C. Navarro-Navarro, A. Gutierrez, E. Sarmiento, A. Troncoso Palacio "Capacidad de Proceso: Una herramienta de decisión Empresarial en el armado de vallas metálicas”, BILO, vol. 2, no. 1, 2020. DOI: http://dx.doi.org/10.17994/bilo

\section{Resumen}

Debido a las frecuentes fluctuaciones económicas que enfrentan las pequeñas empresas del sector metalmecánico, algunas se vuelven insostenibles y otras tienden a desaparecer. Por lo cual, en este documento, se mide la aptitud que tiene un proceso de producción de avisos luminosos con estructuras metálicas, para cumplir con las especificaciones técnicas deseadas. Con la utilización de esta herramienta, se puede medir la capacidad de un proceso, para conocer si es necesario implementar mejoras al sistema tendientes a cumplimiento de los pedidos realizados y a la satisfacción de los clientes, con lo cual se podrá mantener la calidad de la organización y la calidad en la fabricación de los productos, además sirve como aporte a la literatura de este sector industrial.

Palabras claves: Cartas de control XR, Capacidad de Proceso, Control Estadístico del Proceso. 


\begin{abstract}
Due to the frequent economic fluctuations that small companies in the metalworking sector face, some become unsustainable and others tend to disappear. Therefore, in this document, the suitability of a process for the production of light notices with metal structures is measured, to comply with the desired technical specifications. With the use of this tool, the capacity of a process can be measured, to know if it is necessary to implement improvements to the system aimed at fulfilling the orders placed and customer satisfaction, with which the quality of the organization can be maintained. and the quality in the manufacture of the products, also serves as a contribution to the literature of this industrial sector.
\end{abstract}

Keywords: XR control charts, process capacity and statistical process control.

\title{
I. INTRODUCCIÓN
}

La herramienta de estudio que fue utilizada en este proyecto es la carta de control y los diferentes cálculos de capacidad, con el principio y/o finalidad de establecer a través de procedimientos estadísticos en el proceso de fabricación de vallas eléctricas con estructura metálica, donde se recopilo la información del área del producto de un cliente, donde la empresa licito y se le dio unas medidas especificadas, y establecer si este proceso productivo que estamos estudiando si es capaz y así determinar si realmente es rentable para la empresa. Aumentar la productividad en las organizaciones es muy importante, lo que resulta muy atrayente a los inversionistas, empresarios y demás [1]. Por ello en este estudio es importante determinar controles estadísticos que nos aseguren que los parámetros que establecen los clientes de la empresa se verán reflejados en el producto final [2].

Dentro de muchas organizaciones existen actividades con bajos porcentajes de rentabilidad o que simplemente no generan valor a sus productos, servicios y procesos, representando desgasto en todos los recursos necesarios en una organización debido al poco cuidado de procesos amparados por actividades de calidad, generando tiempos improductivos, procedimientos indebidos considerándolos motivos de perdida en materiales, dinero, tiempo, espacios y demás recursos, por consiguiente, se debe realizar adecuadamente los estudios en la productividad de la empresa y se deben contemplar todos los procesos productivos dentro de las organizaciones con la finalidad de estandarizarlos. El beneficio que nace a raíz de la identificación y evaluación de los procesos productivos realizados en la empresa ZEBIT S.A.S, en la medición y mejoramiento de los procesos de la productividad utilizando las herramientas estadísticas ya que, el cual ha venido generando pérdidas en dinero, insumos y otros recursos vitales dentro de la organización.

\section{ESTUDIOS PRIMARIOS DE LA LITERATURA}

En este artículo se habla sobre la implementación de las herramientas de calidad para la mejora en el proceso de fabricación y producción de avisos luminosos con estructuras metálicas [1], se aplica de las cartas de control en proceso la verificar su estabilidad y lograr identificar en los momentos de variación que estuvo fuera de indicaciones le proceso [2] . Además [3] aplicando la capacidad de proceso para verificar el grado de aptitud que tiene un proceso para cumplir con las especificaciones técnicas deseadas. Cuando la capacidad de un proceso es alta, se dice que el proceso es capaz, cuando se mantiene estable a lo largo del tiempo, se dice que el proceso está bajo control, cuando no ocurre esto se dice que el proceso no es adecuado para el trabajo o requiere de inmediatas modificaciones. La importancia de los estudios de capacidad describe el desempeño y la capacidad de los procesos considerando los requerimientos del cliente, características propias del proceso y el efecto del paso del tiempo en el proceso. Para la obtención de la capacidad de procesos o Cp. [4] que es la relación entre la tolerancia especificada y la tolerancia natural del proceso o capacidad de proceso. Este índice relaciona la variabilidad propia del proceso con los límites de especificación establecidos para el producto o servicio de este. Cuando se obtiene la capacidad del proceso se verificar si este es capaz o no de suplir las necesidades, esta capacidad se medí mediante los siguientes indicadores.

- $\quad$ Cuando el Cp. > 2 se dice que le proceso es de clase mundial y tiene calidad seis sigmas

- Cuando el CP se encuentra entre $1.33 \leq \mathrm{CP} \leq 2$ se dice que es proceso es de clase 1 y es más que adecuado

- Cuando el CP se encuentra entre $1 \leq \mathrm{Cp}$. $<1.33$ se dice que el proceso es de clase 2 y es adecuado para el trabajo, pero requiere de un control estricto conforme el CP se acerque a 1

- $\quad$ Cuando el CP se encuentra entre $0.67 \leq \mathrm{Cp}$. $\leq 1$ se dice que el proceso es de clase 3 y no es el adecuado para el trabajo, se necesita un análisis del proceso, requiere modificaciones serias para alcanzar una calidad satisfactoria

- Cuando el Cp. $<0.67$ se dice que el proceso no es el adecuado para el trabajo. Requiere de modificaciones serias como lo indican en [5].

Cpk: Es el valor que caracteriza la relación existente entre la medida del proceso y su distancia al límite de especificación, por el cual el proceso dará un mejor resultado menos correcto. Se define el índice Cpk para tener en cuenta el centrado del proceso. La magnitud de $\mathrm{Cpk}$ respecto $\mathrm{Cp}$ es una medida directa de cuan apartado del centro está operando el proceso. 
$\mathrm{Cpk}=\operatorname{Min}(\mathrm{Cpi}, \mathrm{Cps})$

Cuando $\mathrm{Cp}=\mathrm{Cpk}$ proceso centrado en el punto medio de las especificaciones.

Cuando $\mathrm{Cp}>\mathrm{Cpk}$ proceso descentrado.

Sin embargo, Cpk sólo sigue siendo una medida inadecuada del centrado del proceso, ya que para cualquier valor fijo de $\mu$ en el intervalo de LSL a USL Cpk depende inversamente de $\sigma$ y se hace grande cuando $\sigma$ tiende a cero según [6].

\section{METODOLOGÍA}

Para la resolución de la actividad se ubicó y se definió el proceso a estudiar en la actividad de producción de vallas luminosas, para esto se realizó una recolección de datos durante 25 días tomado 5 muestras a diferentes horario del día, lo siguiente es utilizar las herramientas de calidad como lo son la capacidad de procesos y las cartas de control XR, con el fin de obtener una seria de medidas que indiquen de manera si el proceso es capaz de cumplir con la especificaciones del cliente y si se encuentra en control estadístico.

\section{DESARROLLO}

Datos recolectados durante 25 días, en los cuales se recolectaron 5 muestras por días a diferente horario

\begin{tabular}{|c|rrrrr}
\hline Hora/muestra & 1 & 2 & \multicolumn{1}{c}{3} & \multicolumn{1}{c}{4} & \multicolumn{1}{c}{5} \\
\hline DIA 1 & 10,70 & 11,76 & 11,28 & 11,39 & 10,38 \\
DIA 2 & 11,34 & 11,08 & 11,08 & 11,97 & 11,26 \\
DIA 3 & 11,06 & 11,60 & 11,62 & 10,79 & 11,00 \\
DIA 4 & 10,48 & 10,62 & 10,30 & 10,52 & 11,34 \\
DIA 5 & 11,11 & 11,19 & 10,93 & 10,91 & 11,84 \\
DIA 6 & 11,21 & 10,46 & 9,82 & 10,93 & 11,06 \\
DIA 7 & 10,18 & 11,02 & 10,94 & 10,80 & 10,17 \\
DIA 8 & 11,44 & 10,78 & 11,15 & 9,82 & 10,29 \\
DIA 9 & 11,10 & 10,43 & 10,88 & 10,42 & 11,22 \\
DIA 10 & 10,89 & 10,61 & 11,35 & 10,86 & 11,68 \\
DIA 11 & 10,47 & 10,74 & 11,88 & 11,50 & 11,17 \\
DIA 12 & 11,22 & 11,10 & 10,90 & 11,39 & 11,19 \\
DIA 13 & 11,94 & 11,25 & 11,22 & 10,47 & 10,82 \\
DIA 14 & 10,83 & 10,35 & 10,41 & 10,98 & 10,69 \\
DIA 15 & 10,73 & 11,02 & 10,44 & 11,08 & 11,14 \\
DIA 16 & 10,75 & 11,34 & 10,61 & 11,31 & 12,14 \\
DIA 17 & 11,23 & 10,78 & 10,99 & 10,73 & 10,96 \\
DIA 18 & 10,78 & 11,30 & 11,37 & 12,00 & 11,66 \\
DIA 19 & 10,66 & 11,54 & 10,98 & 11,15 & 11,04 \\
DIA 20 & 10,52 & 11,34 & 10,14 & 11,96 & 11,19 \\
DIA 21 & 10,31 & 10,94 & 10,96 & 12,50 & 11,00 \\
DIA 22 & 11,33 & 10,54 & 11,12 & 11,59 & 11,48 \\
DIA 23 & 10,36 & 11,59 & 10,67 & 10,08 & 10,86 \\
DIA 24 & 10,86 & 10,77 & 11,36 & 10,28 & 11,57 \\
DIA 25 & 11,73 & 11,99 & 10,49 & 10,29 & 11,80
\end{tabular}

Tabla 1. Muestras tomadas del proceso

En carta de control X se evidencia que el proceso está bajo control estadístico ya que presenta variaciones comunes, este se encuentra estable y cumple a cabalidad todas las especificaciones que se le piden. 


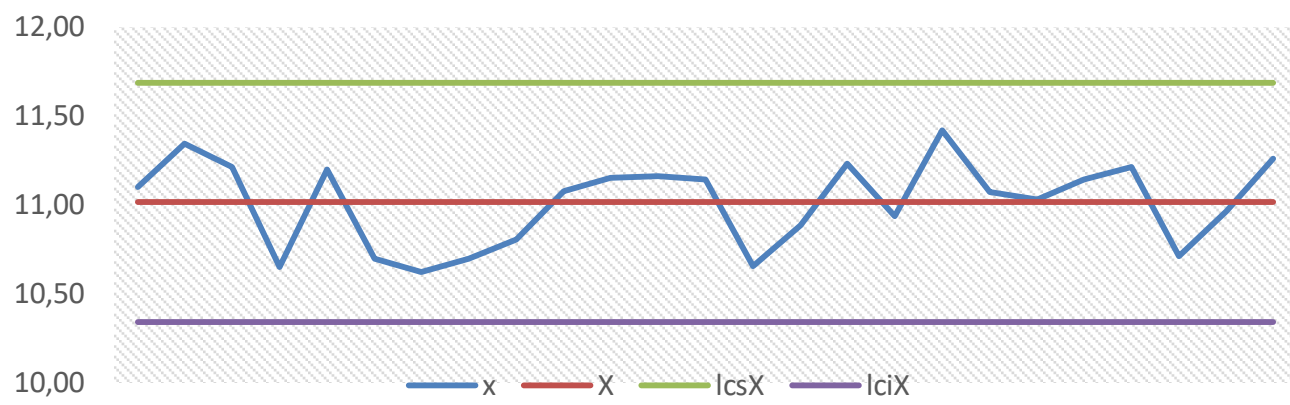

Grafico 1. Variabilidad de la Carta de control X. Fuente: Elaboración propia.

En la carta de control R de lectura individual se analiza que todos los puntos encuentran entre sus límites establecidos, lo que indica que el proceso no sufre variaciones especiales.

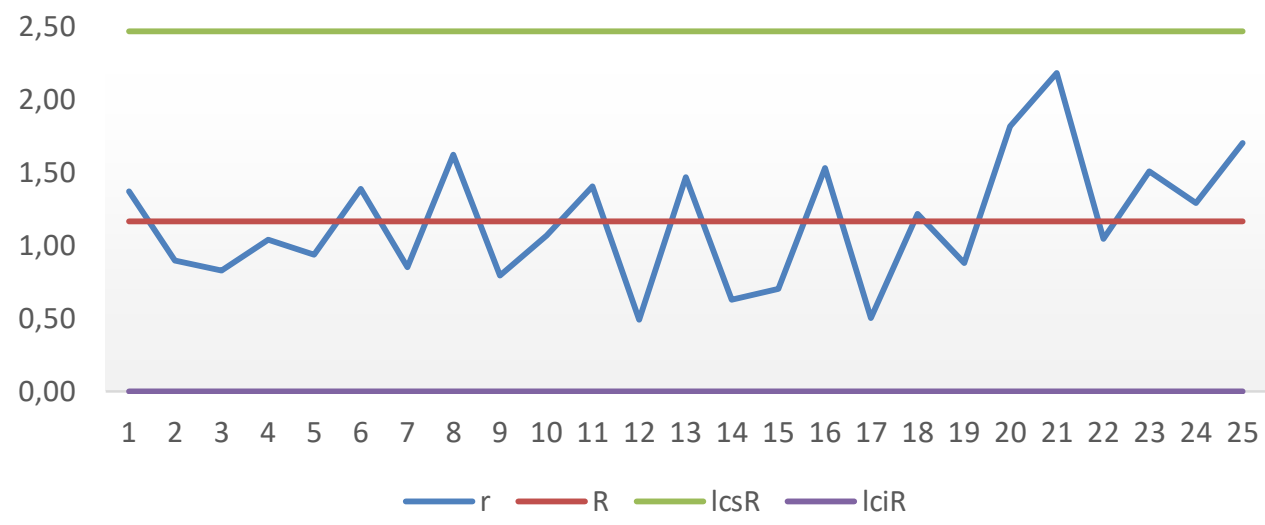

Grafico 2. Variabilidad de la Carta de control R. Fuente: Elaboración propia.

Gráfico de las muestras tomas y las especificaciones de fabricación, en las que se ve en algunos momentos las muestras sobrepasan los límites de control tanto el superior como el inferior. Esto se evidencia en los puntos 13, 25, 26, 28, 50, 61, 77, 93, 96, 105, 116 y 125 que sobre pasaron el límite superior y el límite inferior lo sobrepasan las muestras 7, 56, 83, 98 y 107, esto evidencia que el proceso tiene mucha variabilidad tiende a superar las especificaciones tanto por el límite superior como el inferior

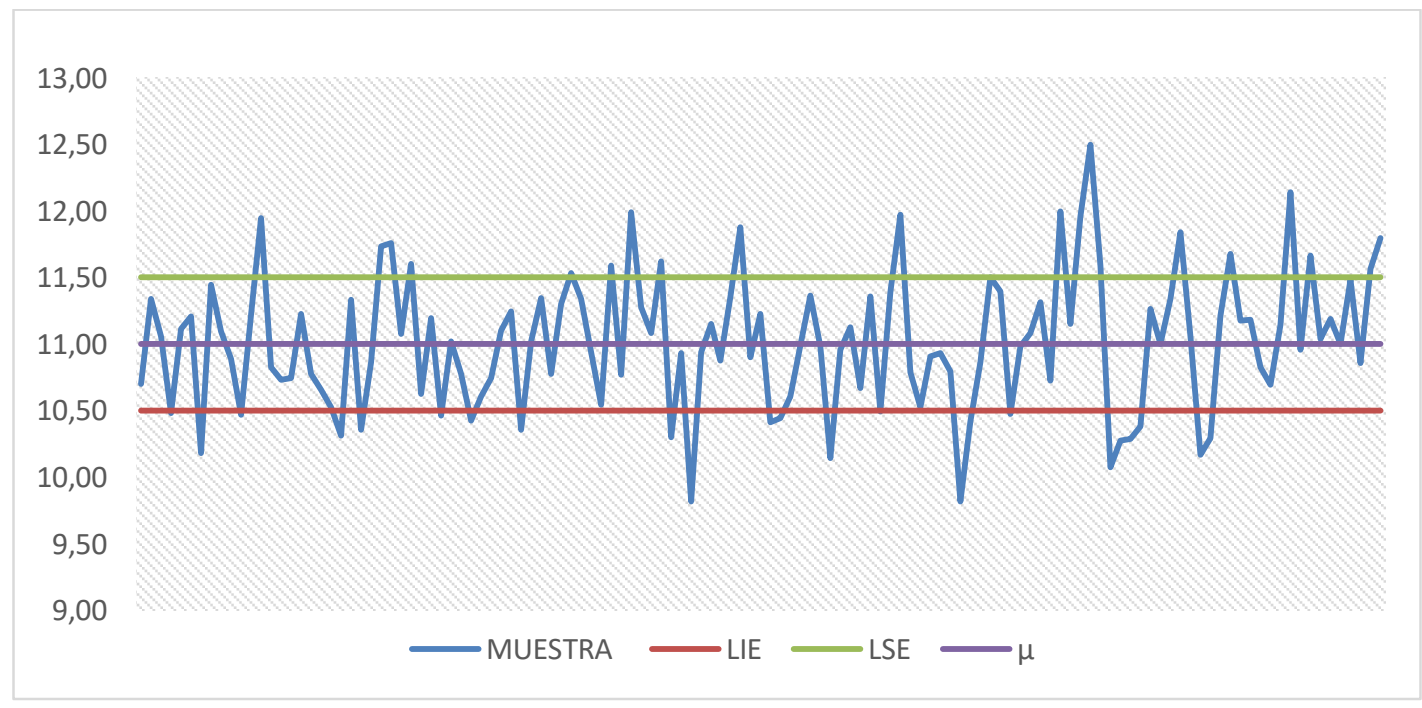

Grafico 3. Variabilidad y tendencia de la Capacidad de proceso. Fuente: Elaboración propia. 
BILO Vol. 2 no. 1, Enero- Junio de 2020

Luego de haber graficado las muestras contra las especificaciones se halló la capacidad del proceso utilizando (1)

$$
C P=\frac{L S E-L I E}{6 * \delta}
$$

Donde los límites son los que le cliente nos especifican y la desviación estándar de la muestra los cuales son 11,5 para límite superior, 10,5 límite inferior y la desviación de 0,505.

Remplazando los datos en (1) de CP se obtiene

$$
C P=\frac{11,5-10,5}{6 * 0,505}=0,330
$$

De se puede deducir que el proceso no es el adecuado para el trabajo. Requiere de modificaciones serias, las cuales podría comenzar con el reajuste de la maquinara para así poder bajar la variabilidad de los datos.

Luego se calcula el Cpk para corroborar la información sobre el proceso, en el cual se utiliza la media de la muestra $(11,1)$, el límite superior de especificación $(11,5)$ y la desviación estándar $(0,505)$ como se denota en $(2)$.

$$
\operatorname{Cpk}(L I E)=\frac{L S E-\bar{X}}{3 * \delta}(2)
$$

Reemplazando en (2) los datos se obtienen

$$
\operatorname{Cpk}(L I E)=\frac{11,5-11,1}{3 * 0,505}=0,264
$$

Se obtuvo que $\mathrm{Cp}$. > Cpk, de este se puede reafirmar que el proceso esta descentralizado y no cumple con especificaciones y en el que las causas especiales de variación aparecen con frecuencia. Produce mala calidad y, además, su desempeño es difícil de pronosticar.

\section{CONCLUSIONES}

Dentro de los resultados se puede concluir que a pesar que el proceso se encuentre en un control estadístico, este no es capaz; ya que el CP calculado no da el rango mínimo para un proceso productivo confiable, lo que se puede hacer es tomar las recomendaciones y establecer el cronograma de actividades lo antes posible. Con los datos encontrados y el porcentaje de no conforme determinado es posible que la licitación sea un trabajo que lleve a perdidas y a déficit económicos para la empresa lo que hace que la empresa se vea mal económicamente. Se recomienda no ejecutar este proceso.

\section{Referencia}

[1] C. Ortiz-Hernandez, A. Troncoso-Palacio, D. Acosta-Toscano, R. Begambre-Meza y B. Troncoso-Mendoza, «Utilización de Herramientas de Calidad para la Mejora en los Procesos de Extrusión de Plásticos,» BILO, vol.

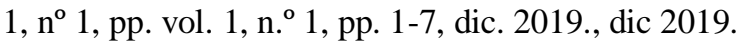

[2] H. Gutieres Pulido y R. De la Vara Salazzr, Control estadistico de la calidad y seis sigma, vol. 2, mexico: mcgraw-hill/interamericana editores, s.a. de c.v, 2009, pp. 183-198.

M.Nuñez, J.Correa, G.Herrera, P.Gómez, S.Morón \& N. Fonseca, “Estudio de percepción sobre energía limpia

[3] y auto sostenible," IJMSOR, vol. 3, no. 1, pp. 11-15, 2018. Recuperado de http://ijmsoridi.com/index.php/ijmsor/article/view/89

[4] C. Hernández Pedrera y F. Da Silva Portofilipe, «Aplicación del control estadístico de procesos (CEP) en el control de su calidad,» scientific electronic library online, vol. 36, n 1 , enero 2016. 
BILO Vol. 2 no. 1, Enero- Junio de 2020

[5] D. Chun y Y. Jung-Gwan, «El uso de metal lleno a través de agujeros para mejorar el aislamiento en LTCC RF y paquetes inalámbricos multichip,» IEEE Transactions on Advanced Packaging , pp. 88 - 99, Febrero 2000.

[6] M. Palacios Lopez y V. Gisber Soler, Control estadistico de la calidad: una aplicacion practica, primera ed., Área de Innovación y Desarrollo,S.L., 2018, pp. 11-85. 